\title{
Cadena de valor de la harina de plátano y su productividad
}

\section{Value chain of banana flour and its productivity}

Cadena de valor

\author{
Nohemí Palacios Cedeño. Mg $^{(1)}$ \\ Jasson Zambrano Montesdeoca. Mg $^{(2)}$ \\ Andy Orozco Quispe. Ing. (3) \\ Ericka Burgos Baque. Ing. ${ }^{(4)}$ \\ ${ }^{1}$ Universidad Técnica de Manabí. Ecuador. email: npalacios@utm.edu.ec. ORCID: https://orcid.org/0000- \\ 0002-0666-5164
}

${ }^{2}$ Universidad Técnica de Manabí. Ecuador. email: jasson.zambrano@utm.edu.ec. ORCID: https://orcid.org/0000-00020501-8918

${ }^{3}$ Universidad Técnica de Manabí. Ecuador. email: dario141994@gmail.com. ORCID: https://orcid.org/00000002-9767-3310

${ }^{4}$ Universidad Técnica de Manabí. Ecuador. email: dulceerikita@live.com

Contacto: dario141994@gmail.com

\section{Resumen}

La cadena de valor es una herramienta empresarial básica para analizar las fuentes de ventaja competitiva, es un medio sistemático que permite examinar todas las actividades que se realizan y sus interacciones. El objetivo de esta investigación fue analizar la cadena de valor de la harina de plátano y su productividad. Los métodos de investigación utilizados fueron el descriptivo, de campo y bibliográfico, el diseño no experimental. La recolección de la información se hizo en el periodo 2018 - 2019, se utilizó la observación directa de los actores de la cadena de valor. Se aplicó la técnica de la encuesta a los productores de la harina de plátano y a los dueños de negocios (tiendas) donde se comercializa el producto. Los resultados evidenciaron que la cadena de valor de la harina de plátano está conformada por las siguientes etapas: proveedores, producción y la comercialización. La productividad en el desarrollo de esta actividad se ha visto afectada negativamente por los siguientes factores: productores con conocimiento empírico y no técnico, falta de recursos económicos, no disponen de maquinarias modernas ni de un proceso de producción estandarizado, deficiencias en la 
distribución del producto, inapropiada ubicación de puntos y centros de venta del producto. Sin embargo, se presentaron como fortalezas la cantidad y calidad de la materia prima y la experiencia del capital humano. En conclusión, la cadena de valor analizada es una fuente de ingresos económicos para la zona. Para incrementar su productividad debe mejorar las debilidades detectadas sustentándose en sus fortalezas.

Palabras clave: cadena de valor, ventaja competitiva, comercialización, productivo

\begin{abstract}
The value chain is a basic business tool to analyze the sources of competitive advantage, it is a systematic means that allows us to examine all the activities carried out and their interactions. The objective of this research was to analyze the value chain of banana flour and its productivity. The research methods used were descriptive, field and bibliographic, non-experimental design. The information was collected in the 2018-2019 period; direct observation of the value chain actors was used. The survey technique was applied to banana flour producers and business owners (stores) where the product is marketed. The results showed that the banana flour value chain is made up of the following stages: suppliers, production and marketing. Productivity in the development of this activity has been negatively affected by the following factors: producers with empirical and non-technical knowledge, lack of economic resources, do not have modern machinery or a standardized production process, deficiencies in product distribution, inappropriate location of points and centers of sale of the product. However, the quantity and quality of the raw material and the experience of human capital were presented as strengths. In conclusion, the value chain analyzed is a source of economic income for the area. To increase your productivity, you must improve the weaknesses detected based on your strengths.
\end{abstract}

Keyword: value chain, competitive advantage, commercialization, productive

\title{
Introducción
}

En la economía ecuatoriana la producción del plátano es una de las actividades agrícolas más significativas dentro del marco local, que por su versatilidad para ser transformado por la industria ha sido reconocido internacionalmente no solo por el volumen de exportación sino también por la calidad del producto. Las provincias más relevantes en cuanto a la producción del plátano son El Oro, Los Ríos, Guayas y Manabí.

A nivel local el plátano es considerado un producto con un alto valor nutritivo, el cual genera una variedad de productos. Uno de ellos es la producción de la harina de plátano, es un emprendimiento que se ha visto involucrado las microempresas, es por ello que llevar el control y la gestión del proceso productivo de esta 
actividad es relativamente importante, generando estrategias de producción, distribución y comercialización que ayuden a incrementar valor al producto. (Correa, 2019)

En el Ecuador el consumo de harina de plátano tiene un porcentaje bajo a diferencia de la de trigo puesto que está en la mente de los consumidores, aquel tipo harina que es más perjudicial para la salud con el tiempo, en cambio el consumo de harina de plátano ayuda a la baja de peso y no incrementar el colesterol malo como lo es el LDL y aumentar el colesterol bueno como es el HDL, en el país se consume harina de plátano en empanadas, bolitas de verde.

En el diagnóstico del sector agropecuario del Ministerio de Agricultura y Ganadería (MAG) señala que en Manabí existen aproximadamente 24 talleres artesanales para la elaboración de la harina de plátano, producto que es comercializado solo dentro del país. En el ámbito económico, social y productivo, la agricultura es una de las actividades más relevantes en la provincia de Manabí y en la parroquia Picoazá no es la excepción, pues muchos emprendedores han buscado en invertir en este rubro el fin de conseguir utilidades para mejorar sus condiciones de vida.

En el año 2005, un grupo de habitantes de la Parroquia pensaron hacer algo productivo y comercial en Picoaza y como había plátanos en Manabí, pensaron en secarla y hacer coladas, se comenzó con un grupo minúsculo de personas, fue así que emprendieron hacer de forma artesanal; se comenzó a pelar a mano, se secaba los plátanos en las terrazas de losa de las casas, una vez secado lo cogían y lo molían a mano, luego lo tamizaban es decir le sacaban las semillas de la harina.

Ese proceso lo hicieron poco tiempo, ya que la voz corrió a personas, instituciones, luego los asociados quisieron dar un gran paso en hacer la asociación más grande, algo industrial, en ese entonces se encontraron con una ayuda del gobierno CADERS (centro de apoyo al desarrollo rural) la cual les proporcionó la infraestructura, maquinaria e insumos necesarios para potenciar sus procesos productivos.

La asociación agro-industrial la Milagrosa en los actuales momentos se dedica a la elaboración de harina de plátano con canela y sin canela, son 34 socios en la empresa, se le puso el nombre de La Milagrosa por el sector que se llama MILAGRO, La materia prima se obtiene de Quevedo, el Carmen donde hay buenos plátanos de calidad, los asociados de La Milagrosa aprovechan los meses donde la materia prima es barata como son los meses de abril- mayo, donde hacen una inversión en comprar y los almacenan con toda las precauciones higiénicas y seguridad.

Saldías, Pineda, \& Helmsing, (2017) definen que las cadenas de valor son todas las actividades encaminadas a llevar un producto desde su concepción hasta su consumo final. Por otra parte, la cadena de valor permite 
identificar y analizar actividades con una importancia estratégica para generar una ventaja competitiva. (Andalucia, 2015)

El termino cadena de valor hace referencia a la vinculación de actividades que una sociedad debe realizar para llevar un producto desde el productor hasta el consumidor final. Las cadenas de valor ayudan a la creación de alianzas productivas, admitiendo el uso más eficiente de los recursos, prevalece el papel de la distribución como factor clave de una mayor competitividad, facilitan el flujo de información entre los actores, mejora el desarrollo de soluciones de una manera conjunta con la identificación de problemas en cada eslabón de la cadena. (Peña, Nieto, \& Díaz, 2008)

En el Ecuador, las organizaciones que se dedican a la comercialización de productos de consumo masivo estiman que la cadena de valor no solo sirve para examinar cada una de los eslabones dentro de la cadena, sino que también permite elevar la eficiencia en el desarrollo de las actividades, poder controlar adecuadamente los costos que incurre la empresa, además de analizar las distintas formas de generar valor a un producto según la demanda del mercado donde incursione la empresa. (Escobar, Cárdenas, \& Aguilar, 2016)

Por lo anteriormente expuesto por varios autores, se establece que la cadena de valor son las actividades de una organización, donde se crea varias relaciones y eslabones con el objetivo de brindar una mejor experiencia al cliente. La cadena de valor es considerada como una herramienta estratégica de contexto interno que nos ayuda a identificar de mejor manera las ventajas competitivas de un negocio frente a un mercado objetivo.

A continuación, se describen los eslabones de la harina de plátano en la" Asociación agro - industrial la Milagrosa":

\section{Recepción de los plátanos}

Una vez recibidos los plátanos se separan los racimos que serán procesados para obtener la harina.

\section{Control de calidad}

Es fundamental revisar que los plátanos que se vayan a utilizar cumplan con las normas de calidad, es decir analizar los mínimos detalles de tamaño, color, buen estado de la fruta, y demás particularidades de la misma, además de que en este punto se determina la altura que tiene el racimo donde los expertos pueden calcular un aproximado de lo que rendirá dicho conjunto.

\section{Cortado (Separación de manos y dedos)}

Esto consiste básicamente en separar el plátano del raquis y las hojas, esto procedimiento es fácil y se lo hace manualmente por lo general los trabajadores utilizan machetes para realizar esta labor, una vez cortados se los pone en bandas transportadoras para realizar el siguiente paso. 


\section{Pelado y troceado}

En este paso se necesita la agilidad de los operarios, debido a que deben proceder manualmente a pelar los plátanos, separando la corteza verde de la blanca, una vez realizado esto colocarlos en la banda transportadora la cual los transporta hasta la máquina cortadora o procesadora para cumplir con el resto del proceso, por lo general esto se hace para controlar el grosor de los pedazos, debido a que si se encuentra muy grande puede producir un retraso en el proceso de secado por lo que este no sería completado y si es muy pequeño al momento de la cocción podría cocinarse demasiado y no tendría valor, según expertos el tamaño apropiado de cortarlos es entre 2 a $4 \mathrm{~mm}$.

\section{Inmersión}

Este proceso se lo realiza en tanques que contienen $1 \%$ de ácido ascórbico y $4 \%$ de ácido cítrico, con el objetivo de que permita evitar la oxidación del fruto por algún cambio de color que se pueda determinar mediante este proceso, luego las rodajas son extraídas con canastillas.

\section{Escurrido}

Se procede a ponerlos en un tanque para completar y darle el tiempo requerido para que se escurran, sacando cualquier residuo de ácido que haya quedado.

\section{Secado}

El fin de este paso es reducir los niveles de humedad con un mínimo restante del 5 al 10\%, para esto se procede a colocar las rodajas que ya han sido escurridas en bandejas de acero inoxidable que se ajustan a la secadora o deshidratadora con una temperatura entre 50 a $65^{\circ} \mathrm{C}$.

\section{Molienda}

Una vez pasado el proceso de secado se lleva los trozos de plátano al departamento de molido, donde se lo deja enfriar y se conduce a trasladarlos al molino de rodillos o de martillos, el cual permite que los dichos trozos sean convertidos en polvo con diminutas y finas partículas, a su vez se echa el saborizante seleccionado que en este caso es de vainilla, dando como resultado el producto final que es la harina.

\section{Tamizado}

A pesar de lo pequeñas que pueden ser las partículas de la harina, es muy probable que queden residuos que no han sido correctamente molidos, por lo que es necesario que pasen por un tamiz para así obtener totalmente un producto de calidad y mucho más fino

\section{Pesado, empacado y sellado}

Luego de que la harina ya ha sido tamizada se procede a llevarla al dosificador micrométrico que permite tener el peso exacto de la cantidad que se colocará en las bolsas de empaque las cuales de preferencia son polipropileno, luego el dosificador las sella herméticamente con el calor, los empaques de las mismas incluyen 
el logo y la información necesaria del producto, por lo general la cantidad requerida en el empaque es de 500 gramos y $1 \mathrm{~kg}$.

\section{Almacenamiento y comercialización}

$\mathrm{Al}$ momento de almacenar se tiene en cuenta requisitos indispensables para mantener la calidad del producto, como son la humedad y altas temperaturas, en el proceso de 46 comercialización se verifica que la transportación sea adecuada para salvaguardar la mercadería, ya que el descuido de estos detalles podría causar el deterioro del producto así se encuentre empaquetado.

En cuanto al análisis de la productividad se define como el valor del producto por unidad de insumo, el cual se deriva de cuatro componentes: la innovación que consiste en un cambio significativo de un producto o servicio en específico; la educación que ayuda a despejar las dudas que se presenta al momento de innovar, generando nuevos conocimientos y habilidades; la eficiencias que permite el correcto manejo de los recursos productivos; y la infraestructura física que permite que los procesos productivos se manejen en un lugar apropiado para el negocio. (Loayza, 2016)

Iquiapaza, (2015) indica que la productividad es una medida de la eficiencia en la que se han combinado y utilizado los recursos para cumplir con los objetivos específicos deseados, en el tiempo programado. Sin embargo, Escobar, Álvarez, Tamayo, \& Correa, (2017) señalan que la productividad se ve relacionado con el factor humano, porque es fuente para generar ventaja competitiva en la organización.

Dentro de las organizaciones se determinan algunos factores para la satisfacción de los clientes como el recurso humano, capital, la tecnología y la materia prima; es imprescindible conocer el desempeño de cada uno de estos puntos y cuanto está aportando cada uno de ellos para el cumplimiento de las metas empresariales. Cuando se analiza las proporciones de los recursos utilizados y se lo compara con los resultados obtenidos estamos hablando de productividad y se los puede relacionar con la eficiencia y eficacia.

Fontalvo, De La Hoz, \& Morelos, (2017) señalan que cuando se habla de productividad se refiere a los procesos en el cual intervienen elementos productivos, para la obtención de un resultado (bien o servicio), cuando hay mejoras, estas se traducen en el hecho que con menos o con los mismos elementos productivos se pueden generar mayor o igual beneficios. Álvarez, (2016) revela que para la generación de productividad es importante analizar estratégicamente: las fortalezas, debilidades, amenazas y oportunidades para identificar las competencias internas y externas de una organización, y a través de este marco estratégico la organización logre superar las barreras competitivas del mercado. 
Sin embargo, dentro de las empresas el análisis estratégico no se está produciendo. Es evidente tal como lo mencionan los autores anteriormente citados en el recorrido teórico realizado en la presente investigación, que la productividad se obtiene mediante la producción de equipo moderno y el mejoramiento de los recursos humanos. Por consiguiente, conviene señalar que el mejoramiento de la productividad o el empleo eficaz de los recursos disponibles es la mejor manera, en realidad la única, de promover el desarrollo futuro en cualquier tipo de empresa. Para medir la productividad en la producción de la harina de plátano se utilizó los siguientes indicadores en función a la información que se obtuvo a través de las fuentes directas (encuestas a productores de harina de plátano, Parroquia Picoaza - Provincia de Manabi):

1.- Productividad Total: Unidades producidas/ insumo empleado

2.- Productividad en función del salario: Unidades producidas/ salario mensual.

3.-Productividad Laboral: Unidades producidas / horas - hombres empleados

4.- Productividad del capital: Salidas / mano de obra + material +energía+ capital + otros.

Atendiendo a las cuestiones expuestas, el objetivo del presente trabajo consiste en analizar la cadena de valor de la harina de plátano y su productividad.

\section{Materiales y métodos}

Para el diseño metodológico de este estudio se utilizó un enfoque cualitativo y cuantitativo, el tipo de investigación fue no experimental, debido a que se observaron los fenómenos en su estado natural para luego ser analizados. Dicha investigación requirió hacer uso de los métodos descriptivo, de campo y bibliográfico que permitieron abordar la realidad de la cadena de valor de la harina de plátano y su productividad durante el periodo de estudio 2018 -2019.

El estudio tuvo una fase documental en la cual se revisaron textos, artículos científicos, tesis relacionadas a las variables de estudio, además de páginas web de organismos oficiales con la finalidad de obtener toda la información necesaria. En la fase de campo, se utilizó la observación directa de los actores de la cadena de valor durante la realización de sus actividades.

Adicionalmente, en la fase cuantitativa la población estuvo determinada por 34 productores afiliados a la Asociación agro - industrial la Milagrosa y 10 dueños de negocios (tiendas) ubicados en un pequeño poblado del Ecuador en la provincia de Manabí en la ciudad de Portoviejo y, para el caso en estudio se aplicó una encuesta a los 34 productores y a los 10 dueños de negocios (tiendas), la mayoría de estas tiendas se encuentran en las zonas rurales de Picoazá. Los datos de esta encuesta se procesaron en Excel, haciendo particular uso de 
la estadística descriptiva que permitió obtener las tablas de frecuencias y gráficos estadísticos de cada una de los ítems del instrumento aplicado.

\section{Resultados}

En la parroquia Picoazá en el sector Milagro se encuentra la asociación "La Milagrosa”, misma que se dedica a la producción de la harina de plátano, dicha producción es alrededor de 400 fundas de $200 \mathrm{Mg}$. El producto se elabora según la cantidad demandada por parte de los dueños de negocios y en temporada alta (temporada escolar) la demanda aumenta. La cantidad de productores (34) que pertenecen a la asociación "La Milagrosa", en conjunto de 10 trabajadores remunerados por el número de horas, perciben un salario de $\$ 6$ dólares por 4 horas de trabajo distribuidos en un horario de 2 horas por la mañana y dos horas por la tarde, su horario de trabajo es una vez al mes. Las comercializaciones del producto se realizan a los diferentes dueños de negocios los cuales son amistades, familia entre otras. Uno de sus puntos fuertes para la comercialización del producto es el comisariato Gonzalo Zambrano, la panadería Rey Pan y la tienda Laz, estas últimas ubicadas en Picoazá. Las maquinas que utilizan en el proceso de producción son 7 en total, los cuales se dividen en dos fases:

Primera fase:

- máquina de pelado y picado

- máquina de secado

Segunda fase:

- molino 1

- molino 2

- máquina de tamizado

- máquina de mezclado

- máquina de embace o sellado

A continuación, se presentan los hallazgos principales, a partir de los resultados de las encuestas aplicadas a los productores de harina de plátano, y así mismo se muestran los indicadores de productividad.

\begin{tabular}{|c|l|c|c|}
\hline N & \multicolumn{1}{|c|}{ Opción de respuesta } & Frecuencia & \% \\
\hline 1 & Pocos proveedores de la materia prima & 9 & $27 \%$ \\
\hline 2 & Falta de financiamiento e inversión & 10 & $29 \%$ \\
\hline 3 & Subida de precios de materia prima & 14 & $41 \%$ \\
\hline 4 & Procesos de producción estandarizado & 1 & $3 \%$ \\
\hline \multicolumn{2}{|c|}{ TOTAL } & $\mathbf{3 4}$ & $\mathbf{1 0 0 \%}$ \\
\hline
\end{tabular}


Tabla 1: Los problemas que se presentan en la producción de la harina de plátano Fuente: Encuesta realizada a los productores

La tabla 1 muestra que los problemas que se presentan en la producción de la harina de plátano son la existencia de pocos proveedores de la materia prima con un $27 \%$, la falta de financiamiento e inversión con el $29 \%$, la subida de precios de la materia prima $41 \%$ y la utilización de procesos de producción estandarizado un $3 \%$. Como se evidencia en los datos la subida de precios de la materia prima es uno de los puntos controversiales al momento de la producción de la harina de plátano, ya que éste varía en función de la temporada de cosecha, problemas invernales, etc; a esto se suma la falta de entidades que le proporcionen crédito y la existencia de pocos proveedores de materia prima, ya que debido a la falta de recursos económicos la asociación la Milagrosa solo cuenta con un proveedor.

\begin{tabular}{|c|l|c|c|}
\hline N & \multicolumn{1}{|c|}{ Opción de respuesta } & Frecuencia & \% \\
\hline 1 & Inestabilidad de los precios & 4 & $12 \%$ \\
\hline 2 & Sobreproducción & 0 & $0 \%$ \\
\hline 3 & Difícil acceso a mercados & 30 & $88 \%$ \\
\hline 4 & Otros TOTAL & 0 & $0 \%$ \\
\hline \multicolumn{2}{|c|}{ TOT } & $\mathbf{3 4}$ \\
\hline
\end{tabular}

Tabla 2: Los factores que afecta a la comercialización del producto Fuente: Encuesta realizada a los productores

En la tabla 2 se aprecia que los factores que afectan a la comercialización del producto es el difícil acceso a los mercados según treinta productores (88\%) mientras que cuatro productores (12\%) mencionan que la inestabilidad en los precios es otro factor que influye negativamente en la comercialización del producto. Llegar a un mercado meta es complicado y mucho más si no se tiene los conocimientos adecuados sobre el tema, para los emprendedores las capacitaciones, seminarios, simposios son de suma importancia para poder entender como comercializar sus productos. En el caso de la asociación "La Milagrosa" de la parroquia de Picoaza, parte del personal no cuentan con los conocimientos para poder comercializar sus productos, además solo cuentan con una moto para poder despachar el producto, lo cual dificulta aún más su proceso de comercialización.

\begin{tabular}{|c|l|c|c|}
\hline N & \multicolumn{1}{|c|}{ Opción de respuesta } & Frecuencia & \% \\
\hline 1 & 15 a 30 días & 8 & $80 \%$ \\
\hline 2 & Mas 1 mes & 2 & $20 \%$ \\
\hline \multicolumn{2}{|c|}{ TOTAL } & $\mathbf{1 0}$ & $\mathbf{1 0 0 \%}$ \\
\hline
\end{tabular}

Tabla3: Tiempo de existencia de la mercadería de harina de plátano en su negocio Fuente: Encuesta realizada a los dueños de negocios 
De acuerdo a los datos que se indican en la tabla 3, el tiempo que disponen del producto la mayoría de negocios (80\%) están en un periodo de tiempo equivalente a 15 a 30 días, un periodo justificado por la demanda que tiene el producto, es relevante mencionar que la mayor parte de negocios se encuentran en las zonas rurales de Picoazá. El producto es conocido en este sector de la población y de igual manera reconocen la marca de la asociación "La Milagrosa”. En cuanto el 20\% restante de los dueños de negocios tienen el producto más de un mes en percha, por la información atribuida a dos locales comerciales que manifestaron que la harina de plátano no se vende con rapidez, porque el producto no tiene una imagen llamativa y no es reconocido a nivel comercial con otras marcas ya posicionadas en el mercado como lo son la Oriental, Banasoya, Vitasoya y Banavit entre otros son de gran competencia ya que está en la mente del consumidor, mientras que la harina de plátano "La Milagrosa" es adquirida una gran parte por dueños de negocios que son familiares, conocidos que conocen o tienen algún tipo de vínculo con algún miembro de la asociación.

\begin{tabular}{|r|l|c|r|}
\hline N & Opción de respuesta & Frecuencia & \multicolumn{1}{c|}{} \\
\hline 1 & Malo & 0 & $0 \%$ \\
\hline 2 & Regular & 1 & $10 \%$ \\
\hline 3 & Bueno & 2 & $20 \%$ \\
\hline 4 & Excelente & 7 & $70 \%$ \\
\hline \multicolumn{2}{|c|}{ TOTAL } & $\mathbf{1 0}$ & $\mathbf{1 0 0 \%}$ \\
\hline
\end{tabular}

Tabla 4: Calidad de la harina de plátano que ofrece la asociación Milagrosa Fuente: Encuesta realizada a los dueños de negocios

Según los resultados que se observan en la tabla 4, el $70 \%$ de los dueños de negocios establecen que el producto es excelente; el $20 \%$ menciona que el producto es bueno, mientras que el $10 \%$ de los dueños de negocios creen que el producto es regular. Para los dueños de negocios en su mayoría les parece que el producto es de muy buena calidad y la califican como excelente (70\%) y bueno (20\%) y la recomienda por los distintos nutrientes que ofrece el producto, Los dueños de negocio mencionan que el producto también lo recomiendan por ser elaborado en la parroquia de Picoaza para ellos es un incentivo promocionar un producto elaborado en la parroquia. Sin embargo, uno de los dueños de negocio cree que el producto es regular (10\%) por la poca creatividad en la presentación del producto.

\section{Indicadores de productividad}

\section{1.- Productividad total:}

Para analizar la productividad total se determinan las cantidades del producto de manera mensual además del valor de los insumos empleados para la elaboración del producto: 
Unidades producidas: 400 fundas mensuales

Insumo empleado: 10 trabajadores cada uno labora 4 horas al mes, el valor por hora es de 1.50 eso quiere decir que ganan 60 dólares mensuales.

$$
P T=\frac{\text { UnidadesProducidas }}{\text { InsumosUtilizados }}=\frac{400}{10}=40 \text { unidades } / \text {-hombres }
$$

El indicador de productividad total por persona es de cuarenta fundas de harina de plátano al día este indicador es relativamente alto. Por la información obtenida por parte de los productores ellos solo laboran un día al mes, si las labores de producción fueran diarias producirán más fundas de harina de plátano con la misma cantidad de personas siendo eficientes y optimizando el tiempo de trabajo, recordemos que la asociación por la demanda limitada no puede generar más unidades para la venta ya que serían recursos y tiempo desperdiciado bajo pedidos por motivos de comercialización se encuentran limitado a mayor escala de producción.

\section{2.- Productividad en función del salario}

Para analizar la productividad en función del salario se determinan las cantidades del producto de manera mensual además del valor de los insumos empleados para la elaboración del producto:

Unidades producidas: 400 fundas mensuales

Insumo empleado: 10 trabajadores cada uno labora 4 horas al mes, el valor por hora es de 1.50 eso quiere decir que un trabajador gana 6 dólares mientras que por los 10 trabajadores ganan 60 dólares mensuales.

$$
P S=\frac{\text { UnidadesProducidas }}{\text { SalarioMensual }}=\frac{400}{60}=6.66 \text { unidades }- \text { salario mes }
$$

La productividad en función del salario es relativamente bajo, ya que por cada 6.66 fundas de harina de plátano ellos perciben 6 dólar de salario por el día que laboran, tomando en cuenta que ellos solo laboran un día al mes, y el costo de venta por unidades están en un rango de $(0.60$ - 0.70 ctvs.) claramente no se evidencia productividad bajo este indicador, ciertamente el negocio cubre el salario de los trabajadores, pero no están generando rentabilidad por el motivo que por cada unidad vendida (0.60-0.70 ctvs.) estarían pagando 1 dólar a sus trabajadores.

3.- Productividad laboral: Unidades producidas / horas - hombres empleados 
Unidades producidas: 400 fundas mensuales

Insumo empleado: 10 trabajadores cada uno labora 4 horas al mes y 48 horas anuales

$$
P L=\frac{\text { Unidadesproducidas }}{\text { hora } * \text { hombres }}=\frac{400}{4 * 10}=\frac{400}{40}=10 \text { unidades por hora }- \text { hombre }
$$

Cada empleado elabora 10 unidades por hora esto es un indicador alto tomando en cuenta que si la asociación laboraría diariamente, la producción por hombre aumentaría considerablemente y así la eficiencia y optimización se reflejaría en el término del proceso productivo al finalizar cada número de fundas de harina de plátano al finalizar en un proceso de producción siempre y cuando la producción fuera diaria, anteriormente se hizo énfasis en la problemática que tiene la asociación "La Milagrosa", factores como la comercialización, la demanda limitada entre otros que producen este tipo de trabas importantes y de suma importancia.

\section{4.- Productividad de capital}

Para analizar la productividad total se determinan las cantidades del producto de manera mensual además el valor de los insumos empleados para la elaboración del producto:

Unidades producidas: 400 fundas mensuales

Insumo empleado: 10 trabajadores cada uno percibe 6 dólares al mes por los 10 trabajadores seria 60 dólares mensuales, materiales serian igual al costo por unidad por el total de unidades elaboradas serían 208 dólares, energía 10 dólares, capital (maquinas) 10000 dólares

$$
\begin{gathered}
P C=\frac{\text { Salidas }}{\text { costomanodeobra }+ \text { material }+ \text { energía }+ \text { capital }+ \text { otros }}=\frac{400}{(60+208+10+10000)} \\
=0.04 \text { fundas por cada unidad de capital }
\end{gathered}
$$

Según los resultados una vez aplicada la formula a lo que se refiere el indicador de capital el resultado es relativamente bajo ya que por cada dólar de capital invertido se obtiene 0.04 fundas de harina de plátano esto quiere decir que el proceso productivo no está generando el $100 \%$ de producción y esto se debe a que la maquinaria y el personal solo laboran 1 solo día al mes, la situación seria distinta si las máquinas y el personal laboraran diariamente, pero la situación actual de la asociación al estar limitados por la falta de generación de nuevos puntos de venta no les permite realizar una mejor gestión en cuanto al proceso productivo.

\section{Discusión}


Por lo anteriormente expuesto, los productores mencionan que su actividad productiva es rentable siempre y cuando la variación del costo de su insumo principal el plátano sea mínimo. Asimismo, dada la necesidad que tienen estos pequeños productores de sobrevivir a corto plazo, junto con las condiciones en las cuales se desarrolla su producción (en relación con el bajo acceso a créditos, maquinarias, asistencia técnica, problemas de comercialización y capacidad de negociación en el mercado), es incoherente proponerles un tipo de producción en el cual los beneficios económicos se generen a mediano y largo plazo, tal como lo proponen los proyectos de desarrollo actuales. Los productores perciben que hay situaciones que están fuera de su control y, por esta razón, tal como lo indica Seligman (1989), no calculan un bienestar económico a largo plazo; en caso de necesitar ayuda en un futuro, la mayoría de ellos acudirían a sus amigos y vecinos apelando a la economía moral o acudirían a la pluriactividad. En relación con los productores con producciones de menor escala, como lo analizado en la presente investigación, los costos de producción siguen siendo muy inciertos, puesto que manifiestan estar expuestos a un sinnúmero de situaciones que no siempre pueden controlar o manejar de manera adecuada, junto con la falta de subsidios por parte del Gobierno. Como menciona Mayer (1999), el subsidio al productor genera utilidades y empleo que se convierten en aumentos en la demanda de productos, y esto tiene efectos multiplicadores en los pequeños productores. Asimismo, los productores de harina de plátano mencionan que existen dificultades frente a la obtención de créditos, esto contribuye a la incertidumbre productiva, ya que por más que ellos quieran tener una producción continua y participar activamente en el mercado capitalista, la fluctuación en los precios de los costos de producción y las dinámicas frente a la estandarización generan dificultades. Esto se debe a que la estandarización de los procesos de producción asume que todos los productores que están participando en estos procesos tienen las mismas capacidades y cuentan con recursos similares, ignorando su heterogeneidad en términos económicos y sociales y dejando de lado la intuición y la experiencia de la vida rural. Para estos productores, el conocimiento vivencial es fundamental y no es lógico plantear manuales estandarizados, porque se estarían ignorando el conocimiento y la experiencia acumulados a lo largo de generaciones. Además, la estandarización sin una adecuada preparación puede conducir a mayores costos e incrementar la vulnerabilidad del productor, pues, como menciona Vélez (2015), reduce la flexibilidad productiva y la autonomía en la toma de decisiones sobre los procesos productivos y de comercialización lo que afecta de manera directa el sustento de los productores. Igualmente, como lo demostraron investigaciones realizadas por Jaffee y Henson (2005) y Shiva (2013), entre otros, estas dinámicas homogeneizantes también afectan la calidad de los productos, la soberanía y el entorno ambiental. De igual manera, la estandarización es un dispositivo que fortalece solamente una visión de mundo, proporcionada por los profesionales que validan ese "estándar" particular (Escobar, 2007). En base a los antecedentes expuestos se debe fortalecer planes y programas comunitarios que consideren factores de apoyo al agricultor con educación, ocupación, equipamiento, comunicación, servicios financieros, transferencia de tecnología para 
proyectos relacionados con la producción y fortalecimiento de la producción e industrialización del principal producto agrícola del País, como es el plátano, y de esta forma potencializar los eslabones de producción y comercialización en la cadena de valor de la harina de plátano, que a su vez les permita ser más productivo y por ende mejorar las condiciones de vida de los productores.

\section{Conclusiones}

Con los resultados obtenidos en la presente investigación se concluye que los actores de la cadena de valor de la harina de plátano en la asociación La Milagrosa, presenta en la etapa de proveedores, variación en los precios de la materia prima según la temporada y pocos proveedores ; en la etapa de producción se evidencian conocimientos empíricos de producción, falta de financiamiento e inversión, no disponen de maquinarias modernas ni de un proceso de producción estandarizado; en la etapa de comercialización se evidencia deficiencias, debido a la existencia de un solo vehículo para la distribución del producto, falta de ubicación de puntos y centros de venta del producto, lo que ha generado que la asociación no pueda expandirse en el mercado local de Picoazá, mucho menos en la ciudad de Portoviejo donde la demanda de este producto puede ser significativa para los productores de la organización.

En relación a los indicadores de productividad como se evidenció en los resultados son moderados, es justificable que estos indicadores no brinden resultados favorables, debido a que el nivel de producción es bajo (400 fundas de harinas al mes) por la falta de puntos de ventas y su vez esto genera inconvenientes en ciertos puntos del proceso productivo. Sin embargo, se presentaron como fortalezas la cantidad, calidad de la materia prima y la experiencia del capital humano. La cadena de valor analizada es una fuente de ingresos económicos para la zona. Para incrementar su productividad debe mejorar las debilidades detectadas sustentándose en sus fortalezas.

\section{Bibliografía}

Álvarez, C. J. (2016). Un modelo de productividad y competitividad para la gestión de operaciones, mercados y Negocios (2594-0163 en línea; 1665-7039 en impreso). Revistas Científicas de la Universidad de Guadalajara.

Andalucía, F. P. (2015). Cadena de valor McKinsey. Obtenido de https://www.andaluciaemprende.es/wpcontent/uploads/2019/02/CADENA-DE-VALOR.pdf 
Correa, A. J. (2019). Diseño de una planta de producción de harina de plátano verde domínico (Musa Cavendishii L.) ecuatoriano mediante secado por aspersión. Obtenido de Repositorio Digital UCE: http://www.dspace.uce.edu.ec/handle/25000/20327

Escobar, F., Cárdenas, E. Y., \& Aguilar, I. (2016). Efectos del Modelo Determinalístico E.O.Q. En Las Comercializadoras de Productos de Consumo Masivo, Milagro - Ecuador. Ciencias Pedagógicas e Innovación.

Escobar, V. J., Álvarez, P. L., Tamayo, C. K., \& Correa, P. K. (2017). Liderazgo informal en las organizaciones: Análisis sobre su impacto e influencia en la productividad y competitividad. Clío América.

Fontalvo, H. T., De La Hoz, G. E., \& Morelos, G. J. (2017). La productividad y sus factores: incidencia en el mejoramiento organizacional. Dimensión Empresarial, 47-60.

Iquiapaza, C. L. (17 de mayo de 2015). Gestión empresarial y su incidencia en la productividad de las microempresas industriales del sector confecciones de ropa de la ciudad de Juliaca, año: 2013 - 2014. Obtenido de Universidad Andina Nestor Cáceres Velasquez. Repositorio de Tesis: http://repositorio.uancv.edu.pe/handle/UANCV/329

Loayza, N. (2016). La productividad como clave del crecimiento y el desarrollo en el Perú y el mundo. . Revista estudios económicos.

Paz, M. R., Pesántez, C. Z., \& Sernaqué, A. J. (2015). El plátano verde en la matriz productiva de la zona 8 de Ecuador. Yachana Revista Científica.

Peña, Y., Nieto, A. P., \& Díaz, R. F. (2008). Cadena de valor: Un enfoque para las agrocadenas. Equidad y Desarrollo, 78-79.

Portter, M. E. (2004). Cadena de valor. Mexico: Editorial CECSA.

Saldías, B. C., Pineda, D. J., \& Helmsing, A. (. (2017). Cadenas de valor y desarrollo local. Una reflexión institucional. En A. (. Helmsing, Universidad y desarrollo regional. Aportes del Cider en sus 40 años (págs. 115-135). Colombia: Ediciones Uniandes.

Unisima. (6 de diciembre de 2017). Plátano Verde: Propiedades veneficios y usos. Obtenido de: https://unisima.com/salud/platano-verde/ 
Seligmann, L. (1989). To be in between: The Cholas as market women. Comparative Studies in Society and History, 31(4), 694-721. Recuperado de:

https://www.jstor.org/stable/179075?seq=1́page_scan_tab_contents.

Mayer, E. (1999). Campesinos y neoliberalismo. Discusión sobre la teoría económica del campesinado con énfasis en el medio andino. Recuperado de: https://www.red.pucp.edu.pe/wpcontent/uploads/biblioteca/160210.pdf

Vélez, L. D. (2015). Adaptabilidad y persistencia de las formas de producción campesinas. Bogotá: Universidad Nacional de Colombia.

Jaffee, S., y Henson, S. (2005). Agro-food exports from developing countries: The challenges posed by standards. En A. M. Aksoy y J. C. Beghin (eds.), Global agricultural trade and developing countries (pp. 91 114). Washington: Banco Mundial.

Shiva, V. (2013). Making peace with the earth. Nueva York: Pluto Press.

Escobar, A. (2007). La invención del tercer mundo. Construcción y deconstrucción. 